International Journal of Pure and Applied Mathematics

Volume $91 \quad$ No. 2 2014, 253-264

ISSN: 1311-8080 (printed version); ISSN: 1314-3395 (on-line version)

url: http://www.ijpam.eu

doi: http://dx.doi.org/10.12732/ijpam.v91i2.12

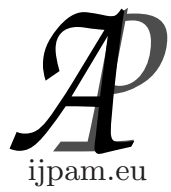

\title{
COMMON FIXED POINT THEOREMS FOR FOUR MAPPINGS IN INTUITIONISTIC FUZZY METRIC SPACES
}

\author{
Saurabh Manro ${ }^{1}$, Shin Min Kang ${ }^{2} \S$ \\ ${ }^{1}$ School of Mathematics and Computer Applications \\ Thapar University \\ Patiala 147004, Punjab, INDIA \\ ${ }^{2}$ Department of Mathematics and RINS \\ Gyeongsang National University \\ Jinju, 660-701, KOREA
}

\begin{abstract}
In this paper, we prove common fixed point theorems for four mappings by using pointwise $R$-weakly commuting and reciprocally continuous mappings satisfying contractive condition in intuitionistic fuzzy metric spaces.
\end{abstract}

AMS Subject Classification: 47H10, 54H25

Key Words: intuitionistic fuzzy metric spaces, reciprocally continuous mappings, pointwise $R$-weakly commuting mappings

\section{Introduction}

Atanassov [3] introduced and studied the concept of intuitionistic fuzzy sets as a generalization of fuzzy sets. In 2004, Park [7] defined the notion of intuitionistic fuzzy metric space with the help of continuous $t$-norms and continuous $t$-conorms. Recently, in 2006, Alaca et al. [2] using the idea of intuitionistic fuzzy sets, defined the notion of intuitionistic fuzzy metric space with the help of continuous $t$-norm and continuous $t$-conorms as a generalization of fuzzy metric space due to Kramosil and Michálek [5]. In 2006, Türkoğlu et al. [9]

Received: December 20, 2013

(c) 2014 Academic Publications, Ltd.

$\S$ Correspondence author url: www.acadpubl.eu 
proved Jungck's common fixed point theorem ([4]) in the setting of intuitionistic fuzzy metric spaces for commuting mappings. For more detail, one can refer to papers $([1],[6],[10],[11])$.

In this paper, we prove a common fixed point theorem for four mappings by using pointwise $R$-weakly commuting and reciprocally continuous mappings satisfying contractive condition in intuitionistic fuzzy metric spaces.

\section{Preliminaries}

Schweizer and Sklar [8] defined the following notions:

Definition 2.1. A binary operation $*:[0,1] \times[0,1] \rightarrow[0,1]$ is continuous $t$-norm if $*$ satisfies the following conditions:

(i) $*$ is commutative and associative;

(ii) $*$ is continuous;

(iii) $a * 1=a$ for all $a \in[0,1]$;

(iv) $a * b \leq c * d$ whenever $a \leq c$ and $b \leq d$ for all $a, b, c, d \in[0,1]$.

Definition 2.2. A binary operation $\diamond:[0,1] \times[0,1] \rightarrow[0,1]$ is continuous $t$-conorm if $\diamond$ satisfies the following conditions:

(i) $\diamond$ is commutative and associative;

(ii) $\diamond$ is continuous;

(iii) $a \diamond 0=a$ for all $a \in[0,1]$;

(iv) $a \diamond b \leq c \diamond d$ whenever $a \leq c$ and $b \leq d$ for all $a, b, c, d \in[0,1]$.

Alaca et al. [2] defined the notion of intuitionistic fuzzy metric space as follows:

Definition 2.3. A 5-tuple $(X, M, N, *, \diamond)$ is said to be an intuitionistic fuzzy metric space if $X$ is an arbitrary set, $*$ is a continuous $t$-norm, $\diamond$ is a continuous $t$-conorm and $M, N$ are fuzzy sets on $X^{2} \times[0, \infty)$ satisfying

(i) $M(x, y, t)+N(x, y, t) \leq 1$ for all $x, y \in X$ and $t>0$;

(ii) $M(x, y, 0)=0$ for all $x, y \in X$;

(iii) $M(x, y, t)=1$ for all $x, y \in X$ and $t>0$ if and only if $x=y$;

(iv) $M(x, y, t)=M(y, x, t)$ for all $x, y \in X$ and $t>0$;

(v) $M(x, y, t) * M(y, z, s) \leq M(x, z, t+s)$ for all $x, y, z \in X$ and $s, t>0$;

(vi) for all $x, y \in X, M(x, y, \cdot):[0, \infty) \rightarrow[0,1]$ is left continuous;

(vii) $\lim _{t \rightarrow \infty} M(x, y, t)=1$ for all $x, y \in X$ and $t>0$;

(viii) $N(x, y, 0)=1$ for all $x, y \in X$;

(ix) $N(x, y, t)=0$ for all $x, y \in X$ and $t>0$ if and only if $x=y$;

(x) $N(x, y, t)=N(y, x, t)$ for all $x, y \in X$ and $t>0$; 
(xi) $N(x, y, t) \diamond N(y, z, s) \geq N(x, z, t+s)$ for all $x, y, z \in X$ and $s, t>0$;

(xii) for all $x, y \in X, N(x, y, \cdot):[0, \infty) \rightarrow[0,1]$ is right continuous;

(xiii) $\lim _{t \rightarrow \infty} N(x, y, t)=0$ for all $x, y \in X$.

Then $(M, N)$ is called an intuitionistic fuzzy metric on $X$. The functions $M(x, y, t)$ and $N(x, y, t)$ denote the degree of nearness and the degree of nonnearness between $x$ and $y$ with respect to $t$, respectively.

Remark 2.4. Every fuzzy metric space $(X, M, *)$ is an intuitionistic fuzzy metric space of the form $(X, M, 1-M, *, \diamond)$ such that $t$-norm $*$ and $t$-conorm $\diamond$ are associated as $x \diamond y=1-((1-x) *(1-y))$ for all $x, y \in X$.

Remark 2.5. In an intuitionistic fuzzy metric space $(X, M, N, *, \diamond)$, $M(x, y, \cdot)$ is non-decreasing and $N(x, y, \cdot)$ is non-increasing for all $x, y \in X$.

Definition 2.6. Let $(X, M, N, *, \diamond)$ be an intuitionistic fuzzy metric space. Then a sequence $\left\{x_{n}\right\}$ in $X$ is said to be

(i) convergent to a point $x \in X$ if

$$
\lim _{n \rightarrow \infty} M\left(x_{n}, x, t\right)=1 \quad \text { and } \quad \lim _{n \rightarrow \infty} N\left(x_{n}, x, t\right)=0
$$

for all $t>0$,

(ii) Cauchy sequence if

$$
\lim _{n \rightarrow \infty} M\left(x_{n+p}, x_{n}, t\right)=1 \text { and } \lim _{n \rightarrow \infty} N\left(x_{n+p}, x_{n}, t\right)=0
$$

for all $t>0$ and $p>0$.

Definition 2.7. An intuitionistic fuzzy metric space $(X, M, N, *, \diamond)$ is said to be complete if and only if every Cauchy sequence in $X$ is convergent.

Türkoğlu et al. [10] defined the following notions:

Definition 2.8. Let $A$ and $S$ be self-mappings of an intuitionistic fuzzy metric space $(X, M, N, *, \diamond)$. Then a pair $(A, S)$ is said to be commuting if

$$
M(A S x, S A x, t)=1 \quad \text { and } \quad N(A S x, S A x, t)=0
$$

for all $x \in X$ and $t>0$.

Definition 2.9. Let $A$ and $S$ be self-mappings of an intuitionistic fuzzy metric space $(X, M, N, *, \diamond)$. Then a pair $(A, S)$ is said to be weakly commuting if

$$
M(A S x, S A x, t) \geq M(A x, S x, t)
$$

and

$$
N(A S x, S A x, t) \leq N(A x, S x, t)
$$

for all $x \in X$ and $t>0$. 
Definition 2.10. Let $A$ and $S$ be self-mappings of an intuitionistic fuzzy metric space $(X, M, N, *, \diamond)$. Then a pair $(A, S)$ is said to be compatible if

$$
\lim _{n \rightarrow \infty} M\left(A S x_{n}, S A x_{n}, t\right)=1 \text { and } \lim _{n \rightarrow \infty} N\left(A S x_{n}, S A x_{n}, t\right)=0
$$

for all $t>0$, whenever $\left\{x_{n}\right\}$ is a sequence in $X$ such that $\lim _{n \rightarrow \infty} A x_{n}=$ $\lim _{n \rightarrow \infty} S x_{n}=u$ for some $u \in X$.

Definition 2.11. ([9]) Let $A$ and $S$ be self-mappings of an intuitionistic fuzzy metric space $(X, M, N, *, \diamond)$. Then a pair $(A, S)$ is said to be pointwise $R$-weakly commuting if given $x \in X$, there exist $R>0$ such that

$$
M(A S x, S A x, t) \geq M(A x, S x, t / R)
$$

and

$$
N(A S x, S A x, t) \leq N(A x, S x, t / R)
$$

for all $t>0$.

Clearly, every pair of weakly commuting mappings is pointwise $R$-weakly commuting with $R=1$.

Definition 2.12. ([6]) Two self-mappings $A$ and $S$ of an intuitionistic fuzzy metric space $(X, M, N, *, \diamond)$ is said to be reciprocally continuous if

$$
A S u_{n} \rightarrow A z \text { and } S A u_{n} \rightarrow S z,
$$

whenever $\left\{u_{n}\right\}$ is a sequence such that $A u_{n} \rightarrow z$ and $S u_{n} \rightarrow z$ for some $z \in X$ as $n \rightarrow \infty$.

If $A$ and $S$ are both continuous, then they are obviously reciprocally continuous, but converse is not true.

Lemma 2.13. ([2], [9]) Let $\left\{u_{n}\right\}$ is a sequence in an intuitionistic fuzzy metric space $(X, M, N, *, \diamond)$. If there exists a constant $k \in(0,1)$ such that

$$
M\left(u_{n}, u_{n+1}, k t\right) \geq M\left(u_{n-1}, u_{n}, t\right)
$$

and

$$
N\left(u_{n}, u_{n+1}, k t\right) \leq N\left(u_{n-1}, u_{n}, t\right)
$$

for $n=1,2,3, \ldots$, then $\left\{u_{n}\right\}$ is a Cauchy sequence in $X$.

Lemma 2.14. ([2], [9]) Let $(X, M, N, *, \diamond)$ be an intuitionistic fuzzy metric space. If there exists a constant $k \in(0,1)$ such that

$$
M(x, y, k t) \geq M(x, y, t) \quad \text { and } \quad N(x, y, k t) \leq N(x, y, t)
$$

for all $x, y \in X$ and $t>0$, then $x=y$. 


\section{Main Results}

For our main theorem, we need the following lemma.

Lemma 3.1. Let $(X, M, N, *, \diamond)$ be a complete intuitionistic fuzzy metric space with $t * t \geq t$ and $(1-t) \diamond(1-t) \leq(1-t)$ for all $t \in[0,1]$. Further, let $A, B, S$ and $T$ be four self-mappings of $X$ satisfying

$(C 1) A(X) \subset T(X)$ and $B(X) \subset S(X)$,

$(C 2)$ there exists a constant $k \in(0,1)$ such that

$$
\begin{aligned}
& {[1+a M(S x, T y, k t)] * M(A x, B y, k t)} \\
& \geq a[M(A x, S x, k t) * M(B y, T y, k t) * M(B y, S x, k t)] \\
& \quad+M(T y, S x, t) * M(A x, S x, t) * M(B y, T y, t) \\
& \quad * M(B y, S x, \alpha t) * M(A x, T y,(2-\alpha) t)
\end{aligned}
$$

and

$$
\begin{aligned}
{[1+a N(S x, T y, k t)] \diamond N(A x, B y, k t) } \\
\leq a[N(A x, S x, k t) \diamond N(B y, T y, k t) \diamond N(B y, S x, k t)] \\
\quad+N(T y, S x, t) \diamond N(A x, S x, t) \diamond N(B y, T y, t) \\
\quad \diamond N(B y, S x, \alpha t) \diamond N(A x, T y,(2-\alpha) t)
\end{aligned}
$$

for all $x, y \in X, a \geq 0, \alpha \in(0,2)$ and $t>0$.

If the pairs $(A, S)$ and $(B, T)$ are pointwise $R$-weakly commuting, then one continuity of the mappings in compatible pair $(A, S)$ or $(B, T)$ implies their reciprocal continuity.

Proof. First, assume that $A$ and $S$ are compatible and $S$ is continuous. We show that $A$ and $S$ are reciprocally continuous. Let $\left\{u_{n}\right\}$ be a sequence such that $A u_{n} \rightarrow z$ and $S u_{n} \rightarrow z$ for some $z \in X$ as $n \rightarrow \infty$. Since $S$ is continuous, we have $S A u_{n} \rightarrow S z$ and $S S u_{n} \rightarrow S z$ as $n \rightarrow \infty$ and since $(A, S)$ is compatible, we have

$$
\lim _{n \rightarrow \infty} M\left(A S u_{n}, S A u_{n}, t\right)=1 \quad \text { and } \lim _{n \rightarrow \infty} N\left(A S u_{n}, S A u_{n}, t\right)=0,
$$

which implies that

$$
\lim _{n \rightarrow \infty} M\left(A S u_{n}, S z, t\right)=1 \text { and } \lim _{n \rightarrow \infty} N\left(A S u_{n}, S z, t\right)=0
$$

for all $t>0$, that is $A S u_{n} \rightarrow S z$ as $n \rightarrow \infty$. By $(C 1)$, for each $n$, there exists $v_{n} \in X$ such that $A S u_{n}=T v_{n}$. Thus, we have $S S u_{n} \rightarrow S z, S A u_{n} \rightarrow S z$, $A S u_{n} \rightarrow S z$ and $T v_{n} \rightarrow S z$ as $n \rightarrow \infty$, whenever $A S u_{n}=T v_{n}$. 
Now we claim that $B v_{n} \rightarrow S z$ as $n \rightarrow \infty$. By $(C 2)$, take $\alpha=1$,

$$
\begin{aligned}
& {\left[1+a M\left(S S u_{n}, T v_{n}, k t\right)\right] * M\left(A S u_{n}, B v_{n}, k t\right)} \\
& \geq a\left[M\left(A S u_{n}, S S u_{n}, k t\right) * M\left(B v_{n}, T v_{n}, k t\right) * M\left(B v_{n}, S S u_{n}, k t\right)\right] \\
& +M\left(T v_{n}, S S u_{n}, t\right) * M\left(A S u_{n}, S S u_{n}, t\right) * M\left(B v_{n}, T v_{n}, t\right) \\
& \quad * M\left(B v_{n}, S S u_{n}, t\right) * M\left(A S u_{n}, T v_{n}, t\right)
\end{aligned}
$$

and

$$
\begin{aligned}
{[1+} & \left.a N\left(S S u_{n}, T v_{n}, k t\right)\right] \diamond N\left(A S u_{n}, B v_{n}, k t\right) \\
\leq & a\left[N\left(A S u_{n}, S S u_{n}, k t\right) \diamond N\left(B v_{n}, T v_{n}, k t\right) \diamond N\left(B v_{n}, S S u_{n}, k t\right)\right] \\
+ & N\left(T v_{n}, S S u_{n}, t\right) \diamond N\left(A S u_{n}, S S u_{n}, t\right) \diamond N\left(B v_{n}, T v_{n}, t\right) \\
& \diamond N\left(B v_{n}, S S u_{n}, t\right) \diamond N\left(A S u_{n}, T v_{n}, t\right) .
\end{aligned}
$$

Taking $n \rightarrow \infty$,

$$
\begin{aligned}
& {[1+a M(S z, S z, k t)] * M\left(S z, B v_{n}, k t\right)} \\
& \geq a\left[M(S z, S z, k t) * M\left(B v_{n}, S z, k t\right) * M\left(B v_{n}, S z, k t\right)\right] \\
& +M(S z, S z, t) * M(S z, S z, t) * M\left(B v_{n}, S z, t\right) \\
& \quad * M\left(B v_{n}, S z, t\right) * M(S z, S z, t)
\end{aligned}
$$

and

$$
\begin{aligned}
{[1+a N(S z, S z, k t)] \diamond N\left(S z, B v_{n}, k t\right) } \\
\leq a\left[N(S z, S z, k t) \diamond N\left(B v_{n}, S z, k t\right) \diamond N\left(B v_{n}, S z, k t\right)\right] \\
+N(S z, S z, t) \diamond N(S z, S z, t) \diamond N\left(B v_{n}, S z, t\right) \\
\quad \diamond N\left(B v_{n}, S z, t\right) \diamond N(S z, S z, t),
\end{aligned}
$$

which implies that

$$
M\left(S z, B v_{n}, k t\right) \geq M\left(B v_{n}, S z, t\right)
$$

and

$$
N\left(S z, B v_{n}, k t\right) \geq N\left(B v_{n}, S z, t\right) .
$$

By Lemma 2.14, we have $B v_{n} \rightarrow S z$ as $n \rightarrow \infty$. Again by $(C 2)$, take $\alpha=1$,

$$
\begin{aligned}
& {\left[1+a M\left(S z, T v_{n}, k t\right)\right] * M\left(A z, B v_{n}, k t\right)} \\
& \geq a\left[M(A z, S z, k t) * M\left(B v_{n}, T v_{n}, k t\right) * M\left(B v_{n}, S z, k t\right)\right] \\
& +M\left(T v_{n}, S z, t\right) * M(A z, S z, t) * M\left(B v_{n}, T v_{n}, t\right) \\
& \quad * M\left(B v_{n}, S z, t\right) * M\left(A z, T v_{n}, t\right)
\end{aligned}
$$


and

$$
\begin{aligned}
{[1+} & \left.a N\left(S z, T v_{n}, k t\right)\right] \diamond N\left(A z, B v_{n}, k t\right) \\
\leq & a\left[N(A z, S z, k t) \diamond N\left(B v_{n}, T v_{n}, k t\right) \diamond N\left(B v_{n}, S z, k t\right)\right] \\
+ & N\left(T v_{n}, S z, t\right) \diamond N(A z, S z, t) \diamond N\left(B v_{n}, T v_{n}, t\right) \\
& \diamond N\left(B v_{n}, S z, t\right) \diamond N\left(A z, T v_{n}, t\right) .
\end{aligned}
$$

Taking $n \rightarrow \infty$,

$$
\begin{aligned}
{[1+} & a M(S z, S z, k t)] * M(A z, S z, k t) \\
\geq & a[M(A z, S z, k t) * M(S z, S z, k t) * M(S z, S z, k t)] \\
& +M(S z, S z, t) * M(A z, S z, t) * M(S z, S z, t) \\
& * M(S z, S z, t) * M\left(A z, T v_{n}, t\right)
\end{aligned}
$$

and

$$
\begin{aligned}
{[1+} & a N(S z, S z, k t)] \diamond N(A z, S z, k t) \\
\leq & a[N(A z, S z, k t) \diamond N(S z, S z, k t) \diamond N(S z, S z, k t)] \\
+ & N(S z, S z, t) \diamond N(A z, S z, t) \diamond N(S z, S z, t) \\
\quad & \diamond N(S z, S z, t) \diamond N(A z, S z, t),
\end{aligned}
$$

which implies that

$$
M(A z, S z, k t) \geq M(A z, S z, t) \quad \text { and } \quad N(A z, S z, k t) \geq N(A z, S z, t) .
$$

By Lemma 2.14, $A z=S z$. Therefore, $S A u_{n} \rightarrow S z$ and $A S u_{n} \rightarrow S z=A z$ as $n \rightarrow \infty$. Hence, $A$ and $S$ are reciprocally continuous on $X$.

Similarly, if the pair $(B, T)$ is compatible and $T$ is continuous, then the proof is similar. This completes the proof.

Now, we prove main theorem.

Theorem 3.2. Let $(X, M, N, *, \diamond)$ be a complete intuitionistic fuzzy metric space with $t * t \geq t$ and $(1-t) \diamond(1-t) \leq(1-t)$ for all $t \in[0,1]$. Further, let $A, B, S$ and $T$ be four self-mappings of $X$ satisfying $(C 1)$ and $(C 2)$. If the pairs $(A, S)$ and $(B, T)$ are pointwise $R$-weakly commuting and one of the mappings in compatible pair $(A, S)$ or $(B, T)$ is continuous, then $A, B, S$ and $T$ have a unique common fixed point.

Proof. By $(C 1)$ since $A(X) \subset T(X)$, for any point $x_{0} \in X$, there exists a point $x_{1} \in X$ such that $A x_{0}=T x_{1}$. Since $B(X) \subset S(X)$, for this point $x_{1} \in X$, we can choose a point $x_{2}$ in $X$ such that $B x_{1}=S x_{2}$ and so on. Inductively, we can define a sequence $\left\{y_{n}\right\}$ in $X$ such that for $n=0,1,2, \ldots$

$$
y_{2 n}=A x_{2 n}=T x_{2 n+1} \quad \text { and } \quad y_{2 n+1}=B x_{2 n+1}=S x_{2 n+2} .
$$


By $(C 2)$, for all $t>0$ and $\alpha=1-q$ with $q \in(0,1)$, we have

$$
\begin{aligned}
& {\left[1+a M\left(y_{2 n}, y_{2 n+1}, k t\right) * M\left(y_{2 n+1}, y_{2 n+2}, k t\right)\right.} \\
& \geq a\left[M\left(y_{2 n+2}, y_{2 n+1}, k t\right) * M\left(y_{2 n+1}, y_{2 n}, k t\right) * M\left(y_{2 n+1}, y_{2 n+1}, k t\right)\right] \\
& \quad+M\left(y_{2 n}, y_{2 n+1}, t\right) * M\left(y_{2 n+2}, y_{2 n+1}, t\right) * M\left(y_{2 n+1}, y_{2 n}, t\right) \\
& \quad * M\left(y_{2 n+1}, y_{2 n+1},(1-q) t\right) * M\left(y_{2 n+2}, y_{2 n},(1+q) t\right) \\
& \geq a\left[M\left(y_{2 n+2}, y_{2 n+1}, k t\right) * M\left(y_{2 n+1}, y_{2 n}, k t\right)\right] \\
& \quad+M\left(y_{2 n}, y_{2 n+1}, t\right) * M\left(y_{2 n+2}, y_{2 n+1}, t\right) * M\left(y_{2 n}, y_{2 n+1}, q t\right)
\end{aligned}
$$

and

$$
\begin{aligned}
{[1+} & \left.a N\left(y_{2 n}, y_{2 n+1}, k t\right)\right] \diamond N\left(y_{2 n+1}, y_{2 n+2}, k t\right) \\
\leq & a\left[N\left(y_{2 n+2}, y_{2 n+1}, k t\right) \diamond N\left(y_{2 n+1}, y_{2 n}, k t\right) \diamond N\left(y_{2 n+1}, y_{2 n+1}, k t\right)\right] \\
& +N\left(y_{2 n}, y_{2 n+1}, t\right) \diamond N\left(y_{2 n+2}, y_{2 n+1}, t\right) \diamond N\left(y_{2 n+1}, y_{2 n}, t\right) \\
& \diamond N\left(y_{2 n+1}, y_{2 n+1},(1-q) t\right) \diamond N\left(y_{2 n+2}, y_{2 n},(1+q) t\right) \\
\leq & a\left[M\left(y_{2 n+2}, y_{2 n+1}, k t\right) \diamond M\left(y_{2 n+1}, y_{2 n}, k t\right)\right] \\
\quad+ & M\left(y_{2 n}, y_{2 n+1}, t\right) \diamond M\left(y_{2 n+2}, y_{2 n+1}, t\right) \diamond M\left(y_{2 n}, y_{2 n+1}, q t\right) .
\end{aligned}
$$

Thus it follows that

$$
\begin{gathered}
M\left(y_{2 n+1}, y_{2 n+2}, k t\right) \geq M\left(y_{2 n}, y_{2 n+1}, t\right) * M\left(y_{2 n+1}, y_{2 n+2}, t\right) \\
* M\left(y_{2 n}, y_{2 n+1}, q t\right)
\end{gathered}
$$

and

$$
\begin{aligned}
N\left(y_{2 n+1}, y_{2 n+2}, k t\right) \leq N\left(y_{2 n}, y_{2 n+1}, t\right) \diamond N\left(y_{2 n+1}, y_{2 n+2}, t\right) \\
\diamond N\left(y_{2 n}, y_{2 n+1}, q t\right) .
\end{aligned}
$$

Since the $t$-norm and the $t$-conorm are continuous and $M(x, y, \cdot)$ is left continuous and $N(x, y, \cdot)$ is right continuous. Letting $q \rightarrow 1$ in (3.1) and (3.2), we have

$$
M\left(y_{2 n+1}, y_{2 n+2}, k t\right) \geq M\left(y_{2 n}, y_{2 n+1}, t\right) * M\left(y_{2 n+1}, y_{2 n+2}, t\right)
$$

and

$$
N\left(y_{2 n+1}, y_{2 n+2}, k t\right) \leq N\left(y_{2 n}, y_{2 n+1}, t\right) \diamond N\left(y_{2 n+1}, y_{2 n+2}, t\right) .
$$

Similarly, we also have

$$
M\left(y_{2 n+2}, y_{2 n+3}, k t\right) \geq M\left(y_{2 n+1}, y_{2 n+2}, t\right) * M\left(y_{2 n+2}, y_{2 n+3}, t\right)
$$

and

$$
N\left(y_{2 n+2}, y_{2 n+3}, k t\right) \leq N\left(y_{2 n+1}, y_{2 n+2}, t\right) \diamond N\left(y_{2 n+2}, y_{2 n+3}, t\right)
$$


In general, for $m=1,2,3, \ldots$

$$
M\left(y_{m+1}, y_{m+2}, k t\right) \geq M\left(y_{m}, y_{m+1}, t\right) * M\left(y_{m+1}, y_{m+2}, t\right)
$$

and

$$
N\left(y_{m+1}, y_{m+2}, k t\right) \leq N\left(y_{m}, y_{m+1}, t\right) \diamond N\left(y_{m+1}, y_{m+3}, t\right) .
$$

Consequently, it follows that for $m=1,2,3, \ldots$ and $p=1,2,3, \ldots$

$$
M\left(y_{m+1}, y_{m+2}, k t\right) \geq M\left(y_{m}, y_{m+1}, t\right) * M\left(y_{m+1}, y_{m+2}, t / k^{p}\right)
$$

and

$$
N\left(y_{m+1}, y_{m+2}, k t\right) \leq N\left(y_{m}, y_{m+1}, t\right) \diamond N\left(y_{m+1}, y_{m+3}, t / k^{p}\right) .
$$

As $p \rightarrow \infty$,

$$
M\left(y_{m+1}, y_{m+2}, k t\right) \geq M\left(y_{m}, y_{m+1}, t\right)
$$

and

$$
N\left(y_{m+1}, y_{m+2}, k t\right) \leq N\left(y_{m}, y_{m+1}, t\right) .
$$

Hence by Lemma 2.13, $\left\{y_{n}\right\}$ is a Cauchy sequence in $X$. Since $X$ is complete, $\left\{y_{n}\right\}$ converges to $z \in X$. Its subsequences $\left\{A x_{2 n}\right\},\left\{T x_{2 n+1}\right\},\left\{B x_{2 n+1}\right\}$ and $\left\{S x_{2 n+2}\right\}$ also converges to $z$.

Now, suppose that $(A, S)$ is a compatible pair and $S$ is continuous. Then by Lemma 3.1, $A$ and $S$ are reciprocally continuous, thus $S A x_{n} \rightarrow S z$ and $A S x_{n} \rightarrow A z$ as $n \rightarrow \infty$. As $(A, S)$ is a compatible pair, we have

$$
\lim _{n \rightarrow \infty} M\left(A S x_{n}, S A x_{n}, t\right)=1 \quad \text { and } \quad \lim _{n \rightarrow \infty} N\left(A S x_{n}, S A x_{n}, t\right)=0,
$$

that is,

$$
M(A z, S z, t)=1 \quad \text { and } \quad N(A z, S z, t)=0 .
$$

Hence $A z=S z$. Since $A(X) \subset T(X)$, there exists a point $p \in X$ such that $A z=T p=S z$. By $(C 2)$, take $\alpha=1$,

$$
\begin{aligned}
& {[1+a M(S z, T p, k t)] * M(A z, B p, k t)} \\
& \geq a[M(A z, S z, k t) * M(B p, T p, k t) * M(B p, S z, k t)] \\
& \quad+M(T p, S z, t) * M(A z, S z, t) * M(B p, T p, t) \\
& \quad * M(B p, S z, t) * M(A z, T p, t)
\end{aligned}
$$

and

$$
\begin{aligned}
{[1+a N(S z, T p, k t)] \diamond N(A z, B p, k t) } \\
\leq a[N(A z, S z, k t) \diamond N(B p, T p, k t) \diamond N(B p, S z, k t)] \\
+N(T p, S z, t) \diamond N(A z, S z, t) \diamond N(B p, T p, t) \\
\quad \diamond N(B p, S z, t) \diamond N(A z, T p, t),
\end{aligned}
$$


which implies that

$$
M(A z, B p, k t) \leq M(A z, B p, t) \quad \text { and } \quad N(A z, B p, k t) \geq N(A z, B p, t)
$$

for all $t>0$. By Lemma 2.14, we have $A z=B p$. Thus, $A z=B p=S z=T p$. Since $A$ and $S$ are pointwise $R$-weakly commuting mappings, there exists $R>0$ such that

$$
M(A S z, S A z, t) \geq M(A z, S z, t / R)=1
$$

and

$$
N(A S z, S A z, t) \leq N(A z, S z, t / R)=0 .
$$

Therefore, $A S z=S A z$ and $A A z=A S z=S A z=S S z$.

Similarly, $B$ and $T$ are pointwise $R$-weakly commuting mappings, we have $B B p=B T p=T B p=T T p$. Again by $(C 2)$, take $\alpha=1$,

$$
\begin{aligned}
& {[1+a M(S A z, T p, k t)] * M(A A z, B p, k t)} \\
& \geq a[M(A A z, S A z, k t) * M(B p, T p, k t) * M(B p, S A z, k t)] \\
& \quad+M(T p, S A z, t) * M(A A z, S A z, t) * M(B p, T p, t) \\
& \quad * M(B p, S A z, t) * M(A A z, T p, t)
\end{aligned}
$$

and

$$
\begin{aligned}
{[1+} & a N(S A z, T p, k t)] \diamond N(A A z, B p, k t) \\
\leq a & N(A A z, S A z, k t) \diamond N(B p, T p, k t) \diamond N(B p, S A z, k t)] \\
+ & N(T p, S A z, t) \diamond N(A A z, S A z, t) \diamond N(B p, T p, t) \\
& \diamond N(B p, S A z, t) \diamond N(A A z, T p, t),
\end{aligned}
$$

which implies that

$$
M(A A z, A z, k t) \leq M(A A z, A z, t)
$$

and

$$
N(A A z, A z, k t) \geq N(A A z, A z, t) .
$$

Hence by Lemma 2.14, $A A z=A z=S A z$. Hence $A z$ is common fixed point of $A$ and $S$. Similarly by $(C 2), B p=A z$ is a common fixed point of $B$ and $T$. Hence, $A z$ is a common fixed point of $A, B, S$ and $T$.

Finally, suppose that $A p(\neq A z)$ is another common fixed point of $A, B, S$ and $T$. Again by $(C 2)$, take $\alpha=1$,

$$
\begin{aligned}
& {[1+a M(S A z, T A p, k t)] * M(A A z, B A p, k t)} \\
& \geq a[M(A A z, S A z, k t) * M(B A p, T A p, k t) * M(B A p, S A z, k t)] \\
& +M(T A p, S A z, t) * M(A A z, S A z, t) * M(B A p, T A p, t) \\
& \quad * M(B A p, S A z, t) * M(A A z, T A p, t)
\end{aligned}
$$


and

$$
\begin{aligned}
{[1+a N(S A z, T A p, k t)] \diamond N(A A z, B A p, k t) } \\
\leq a[N(A A z, S A z, k t) \diamond N(B p, T A p, k t) \diamond N(B A p, S A z, k t)] \\
+\quad N(T A p, S A z, t) \diamond N(A A z, S A z, t) \diamond N(B A p, T A p, t) \\
\quad \diamond N(B A p, S A z, t) \diamond N(A A z, T A p, t),
\end{aligned}
$$

which implies that

$$
M(A z, A p, k t) \geq M(A z, A p, t) \quad \text { and } \quad N(A z, A p, k t) \leq N(A z, A p, t) .
$$

Hence by using Lemma 2.14, $A z=A p$. Thus uniqueness follows. This completes the proof.

If $S$ and $T$ are identity mappings in Theorem 3.2, we get following result.

Corollary 3.3. Let $(X, M, N, *, \diamond)$ be a complete intuitionistic fuzzy metric space with $t * t \geq t$ and $(1-t) \diamond(1-t) \leq(1-t)$ for all $t \in[0,1]$. Further, let $A$ and $B$ be reciprocally continuous mappings on $X$ satisfying

$(C 3)$ there exists a constant $k \in(0,1)$ such that

$$
\begin{aligned}
& {[1+a M(x, y, k t)] * M(A x, B y, k t)} \\
& \geq a[M(A x, x, k t) * M(B y, y, k t) * M(B y, x, k t)] \\
& \quad+M(y, x, t) * M(A x, x, t) * M(B y, y, t) \\
& \quad * M(B y, x, \alpha t) * M(A x, y,(2-\alpha) t)
\end{aligned}
$$

and

$$
\begin{aligned}
& {[1+a N(x, y, k t)] \diamond N(A x, B y, k t)} \\
& \leq a[N(A x, x, k t) \diamond N(B y, y, k t) \diamond N(B y, x, k t)] \\
& \quad+N(y, x, t) \diamond N(A x, x, t) \diamond N(B y, y, t) \\
& \quad \diamond N(B y, x, \alpha t) \diamond N(A x, y,(2-\alpha) t)
\end{aligned}
$$

for all $x, y \in X, a \geq 0, \alpha \in(0,2)$ and $t>0$.

Then $A$ and $B$ has a unique common fixed point.

\section{References}

[1] C. Alaca, I. Altun, D. Türkoğlu, On compatible mappings of type (I) and (II) in intuitionistic fuzzy metric spaces, Commun. Korean Math. Soc., 23, No. 3 (2008), 427-446, doi: 10.4134/CKMS.2008.23.3.427 
[2] C. Alaca, D. Türkoğlu, C. Yildiz, Fixed points in intuitionistic fuzzy metric spaces, Chaos, Solitons Fractals, 29, No. 5 (2006), 1073-1078, doi: 10.1016/j.chaos.2005.08.066

[3] K.T. Atanassov, Intuitionistic fuzzy sets, Fuzzy Sets and System, 20, No. 1 (1986), 87-96, doi: 10.1016/S0165-0114(86)80034-3

[4] G. Jungck, Commuting mappings and fixed points, Amer. Math. Monthly, 83, No. 4 (1976), 261-263, doi: 10.2307/2318216

[5] I. Kramosil, J. Michálek, Fuzzy metric and statistical metric spaces, Kybernetika, 11, No. 5 (1975), 326-334, doi: 10.2298/FIL0802043K

[6] S. Muralisankar, G. Kalpana, Common fixed point theorems in intuitionistic fuzzy metric spaces using new contractive condition of integral type, Int. J. Contemp. Math. Sci., 4, No. 11 (2009), 505-518.

[7] J.H. Park, Intuitionistic fuzzy metric spaces, Chaos, Solitons Fractals, 22, No. 5 (2004), 1039-1046, doi: 10.1016/j.chaos.2004.02.051

[8] B. Schweizer, A. Sklar, Probabilistic Metric Spaces, North Holland, Amsterdam, 1983.

[9] D. Türkoğlu, C. Alaca, Y.J. Cho, C. Yildiz, Common fixed point theorems in intuitionistic fuzzy metric spaces, J. Appl. Math Comput., 22, No. 1-2 (2006), 411-424, doi: 10.1007/BF02896489

[10] D. Türkoğlu, C. Alaca, C. Yildiz, Compatible maps and compatible maps of type $(\alpha)$ and $(\beta)$ in intuitionistic fuzzy metric spaces, Demonstratio Math., 39, No. 3 (2006), 671-684.

[11] D. Türkoğlu, C. Alaca, C. Yildiz, Common fixed point theorems of compatible maps in intuitionistic fuzzy metric spaces, Southeast Asian Bull. Math., 32, No. 1 (2008), 21-33. 